\title{
Adhesion between dendritic cells and epithelial cells maintains the gut barrier during bacterial sampling
}

\section{J E Collins}

A new mechanism for bacterial uptake in mucosal tissues is proposed that is mediated by dendritic cells endowed with the ability to make intercellular adhesive links directly with epithelial cells thereby preserving the integrity of the gut barrier

$\mathrm{T}$ he intimate nature of the cellular interactions that occur in the gut epithelium where the mucosal immune system interfaces with the external environment is the subject of this fascinating paper by Rescigno et al. Dendritic cells (DCs ) are arguably the most important cell type in the immune system. Although bewilderingly heterogeneous in phenotype and function, they are the only antigen presenting cell type capable of presenting peptides to virgin $\mathrm{T}$ cells, thereby initiating cell mediated immunity to newly encountered antigens. Given the delicate balance which exists in the gut between $\mathrm{T}$ cell tolerance and responsiveness to luminal antigens (a breakdown of which is likely to be responsible for food sensitive enteropathies and inflammatory bowel disease), DCs are probably key factors in controlling $\mathrm{T}$ cell responses in the gut wall. DCs are present in all organised lymphoid tissues and an earlier report in rats suggested that they might also be present in the gut epithelium. ${ }^{1}$ This paper presents evidence for a new and exciting mechanism of antigen sampling as it demonstrates for the first time that DCs can penetrate the epithelium to directly take up bacteria from the gut lumen without compromising the barrier function of the gut. This mechanism is facilitated because DCs appear to be endowed with the ability to make homotypic intercellular adhesive links directly with gut epithelial cells.

The gut mucosa is lined by a layer of epithelial cells that form a barrier between the host and the external environment. Epithelial cells are specialised to perform this role because they are polarised and possess distinct adhesion mechanisms for this purpose (see fig 1). ${ }^{2}$ Tight junctions at the apicolateral surface maintain a permeability seal and act as a fence for proteins in the membrane, and together with adherens junctions they are linked to the actin cytoskeleton..$^{3-5}$ Desmosomes act as rivets between cells connecting the keratin filament system thus providing tissue cohesion and strength. ${ }^{67}$ Rescigno et al show that DCs express the tight junction proteins occludin, claudin 1 , and $\mathrm{ZO}-1$ and provide evidence for tight junction-

Rescigno M, Urbano M, Valzasina B, et al. Dendritic cells express tight

junction proteins and penetrate gut epithelial monolayers to sample bacteria. Nat Immunol $2001 ; 2: 288-90$

Penetration of the gut mucosa by pathogens expressing invasion genes is believed to occur mainly through specialized epithelial cells, called $M$ cells, that are located in Peyer's patches. However, Salmonella typhimurium that are deficient in invasion genes encoded by Salmonella pathogenicity island 1 (SPI1) are still able to reach the spleen after oral administration. This suggests the existence of an alternative route for bacterial invasion, one that is independent of $M$ cells. We report here a new mechanism for bacterial uptake in the mucosa tissues that is mediated by dendritic cells (DCs). DCs open the tight junctions between epithelial cells, send dendrites outside the epithelium and directly sample bacteria. In addition, because DCs express tight-junction proteins such as occludin, claudin 1 and zonula occludens 1, the integrity of the epithelial barrier is preserved.

like structures between DCs and gut epithelial cells, thus maintaining the permeability barrier. Transepithelial resistance, a measure of the "tightness" of the epithelial barrier, is not altered during DC-epithelial cell interaction and uptake of bacteria. The mechanism used by DCs to interact with yet maintain tight junctions is not understood but may involve interactions with the tight junction transmembrane protein occludin. However, among the interesting discoveries in this paper is the observation that DCs are also shown to express a range of other cell adhesion related molecules, including E-cadherin and $\beta$-catenin, proteins found in the adherens junction. The significance of this is unclear.

It is tempting to speculate that DCs are capable of making other types of adhesive links with mucosal epithelial cells in addition to tight junctions. In fact, the adhesive structures thought to be tight junctions and shown in the electron micrographs in fig 2 of their paper actually resemble junctions of the zonula adherens or desmosome type (compare with fig 1 in this article). One of the defining characteristics of tight junctions, as seen in electron micrographs (fig 1), is occlusion of the junctional intercellular space. ${ }^{2}$ The tight junctionlike structures shown by Rescigno et al are not occluded in the junctional intercellular spaces but rather show the presence of distinct intercellular spaces. In addition, the plaque structures are much more developed and brush like, as in adherens or desmosome junctions. Therefore, DCs may make use of interactions with the basolateral cell membranes of intestinal epithelial cells in order to gain entry to the epithelium, especially as the tight junctions are

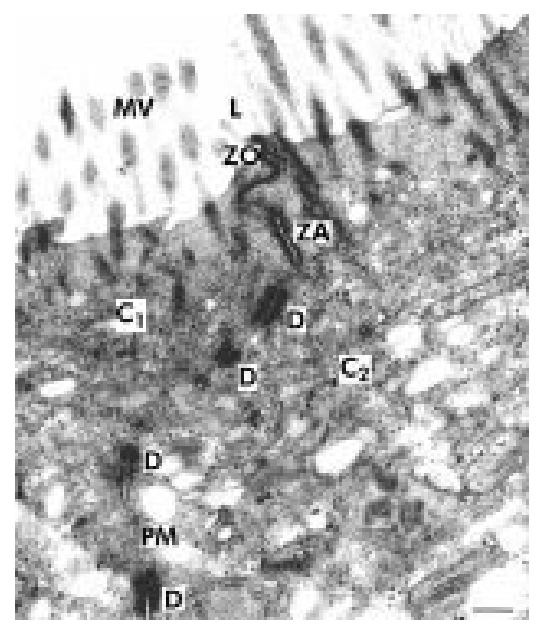

Figure 1 Electron micrograph of the subapical junctional complex of human intestinal epithelium. $C_{1}$ and $C_{2}$ are two adjacent gut enterocytes, each contributing one half to the respective junctions. MV, microvilli; L, lumen; ZO, tight junction showing occlusion of the junctional intercellular space; ZA, zonula adherens with associated actin filaments; $D$, desmosome with associated keratin filaments; PM, plasma membrane. Bar $=0.2 \mu \mathrm{m}$. 
localised at the most apicolateral parts of the cells, someway from the basal parts of the cells.

Extrajunctional E-cadherin and desmosomal cadherins are also known to be expressed in the lateral membranes of epithelial cells ${ }^{5-8}$ and thus may provide molecular handholds for initial points of interaction between the two cell types. In addition, formation and stabilisation of the tight junction is intimately related to the assembly of adherens junctions. ${ }^{9-11}$ DCs have been localised in vivo within the epithelium of normal rat intestine ${ }^{1}$ so they may be routinely anchored there below the tight junctions by other epithelial type cell-cell contacts awaiting a signal to interact with tight junctions, perhaps facilitated by upregulation and redistribution of occludin and claudin. In this context, it may be significant that in these experiments DCs could not interact from the apical side of the epithelium where they would not have access to other epithelial junctional proteins in cells with mature contacts.

Neutrophils can also migrate across epithelia, and occludin influences this process. $^{12}$ When neutrophils interact with epithelium or endothelium they are capable of traversing from the apical side paracellularly as well as from the basolateral side, ${ }^{13}{ }^{14}$ suggesting that they may interact with or influence tight junctions via different mechanisms.

Another notable aspect of this study is the observation that transepithelial resistance (TER) and the structure of tight junctions are not altered during interactions between DCs and epithelial cells.
This absence of tight junction alteration and TER change has also been confirmed in studies of neutrophil migration. ${ }^{14}$ Interestingly, neutrophils have been shown to preferentially migrate across endothelial monolayers at tricellular points of cell contact where tight junction discontinuities are normally observed and this has been proposed as a reason why tight junctions need not be disrupted in this system. ${ }^{14}$ It is unknown whether this phenomenon exists in intestinal epithelia.

This paper therefore provides evidence for a novel and elegant method of bacterial sampling that may represent a very important component of mucosal surveillance, not least because the contribution of the non-Peyer's patch gut epithelium greatly increases the surface available for detection and response to microorganisms. It will be of interest to know whether similar mechanisms exist in other mucosal sites such as the lung where DCs of similar type have been described and, in addition, whether DCs can be used as a mode of entry and spread by pathogenic microorganisms.

\section{Gut 2002;50:449-450}

A..................

\section{Author's affiliation}

J E Collins, Division of Infection, Inflammation and Repair, University of Southampton School of Medicine, Southampton General Hospital, Southampton SO16 6YD, UK; jec3@soton.ac.uk

\section{REFERENCES}

1 Maric I, Holt PG, Perdue MH, et al. Class II $\mathrm{MHC}$ antigen (la)-bearing dendritic cells in the epithelium of the rat intestine. J Immunol 1996;156:1408-14.
2 Farquhar MG, Palade GE. Junctional complexes in various epithelia. J Cell Biol 1963;17:375-412.

3 Collins JE. Classical and desmosomal cadherins. In: Malhotra SK, ed. Greenwich, Connecticut: Jai Press Inc, 1996:75-108.

4 Tsukita $S$, Furuse $M$, Itoh $M$. Structural and signalling molecules come together at tight junctions. Curr Opin Cell Biol 1999; 11:628-33.

5 Yap AS, Brieher WM, Gumbiner BM. Molecular and functional analysis of cadherin-based adherens junctions. Annu Rev Cell Dev Biol 1997; 13:1 19-46.

6 North AJ, Bardsley WG, Hyam J, et al. Molecular map of the desmosomal plaque. J Cell Sci 1999;112(Pt 23):4325-36.

7 Green KJ, Gaudry CA. Are desmosomes more than tethers for intermediate filaments? Nat Rev Mol Cell Biol 2000;1:208-16.

8 Mattey DL, Garrod DR. Calcium-induced desmosome formation in cultured kidney epithelial cells. J Cell Sci 1986;85:95-111.

9 Itoh M, Nagafuchi A, Moroi S, et al. Involvement of ZO-1 in cadherin-based cell adhesion through its direct binding to alpha catenin and actin filaments. J Cell Biol 1997; 138:181-92.

10 Boller K, Vestweber D, Kemler R. Cell-adhesion molecule uvomorulin is localized in the intermediate junctions of adult intestinal epithelial cells. J Cell Biol 1985;100:327-32.

11 Gumbiner B, Stevenson B, Grimaldi A. The role of the cell adhesion molecule uvomorulin in the formation and maintenance of the epithelial junctional complex. J Cell Biol 1988;107: 1575-87.

12 Huber D, Balda MS, Matter K. Occludin modulates transepithelial migration of neutrophils. J Biol Chem 2000;275:5773-8.

13 Colgan SP, Parkos CA, Delp C, et al. Neutrophil migration across cultured intestinal epithelial monolayers is modulated by epithelial exposure to IFN-gamma in a highly polarized fashion. J Cell Biol 1993; 120:785-98.

14 Burns AR, Bowden RA, MacDonell SD, et al. Analysis of tight junctions during neutrophil transendothelial migration. J Cell Sci 2000;113(Pt 1):45-57. 